\title{
The pedagogical device, framework of analysis of the teaching process and evaluation of the reading comprehension
}

\author{
[Le dispositif pédagogique, cadre d'analyse du processus d'enseignement \\ et d'évaluation de la compréhension de l'écrit]
}

\author{
Zineb Haroun
}

DOI: 10.18355/XL.2018.11.01XL.12

\begin{abstract}
This contribution aims at presenting the methodological framework, which enabled a report on the reality of teaching reading comprehension for $5^{\text {th }}$ year pupils, in the Algerian primary schools. We approached the reality based on pedagogical devices, which prove to be an analytical framework and integrator of teaching - learning and an evaluation of reading comprehension due to its systematic character. We practically aim at highlighting its transposition into the didactics of writing field in FFL, going through the constitution of its input, which offers quantitative and qualitative analytical possibilities. The research work is also interested in the teaching practices of reading comprehension and in the pupil's learning as an observational framework of class situations through case studies.
\end{abstract}

Key words: devices, pedagogical, teaching, evaluation, comprehension

\section{Résumé}

Cette contribution a pour objectif de présenter le cadre méthodologique ayant permis de rendre compte de la réalité de l'enseignement de la compréhension de l'écrit en $5^{\mathrm{e}}$ année primaire de l'école algérienne. Cette réalité est approchée à partir du dispositif pédagogique qui s'avère un cadre d'analyse et intégrateur de l'enseignement apprentissage et de l'évaluation de la compréhension de l'écrit du fait de son caractère systémique. Elle tente plus particulièrement de mettre en évidence sa transposition dans le champ de la didactique de l'écrit en FLE en revenant sur le parcours de la constitution de ses entrées qui offrent des possibilités d'analyse à la fois quantitative et qualitative. Il se présente également pour les pratiques d'enseignement de la compréhension de l'écrit et pour les démarches d'apprentissage des élèves comme un cadre d'observation des situations de classe à travers les études de cas.

Mots-clés : dispositif, pédagogique, enseignement, évaluation, compréhension

\section{Le contexte d'introduction du dispositif pédagogique}

L'adoption du dispositif pédagogique comme cadre d'observation de la compréhension de l'écrit en contexte scolaire algérien prend son origine dans la revue de la littérature qui révèle des rapports d'interdépendance entre son enseignement, son apprentissage et son évaluation. Ces rapports rendent compte du caractère complexe des variables intervenant dans le cadre du processus d'enseignement/apprentissage et d'évaluation et de leurs interrelations. Celles-ci s'établissent entre des apprenants et un enseignant dont les actions convergent vers l'atteinte d'objectifs d'enseignement et d'apprentissage. Les rapports existant entre ces acteurs ainsi que les objectifs font partie du dispositif (Montandon, 2002) qui a justement pour fonction « de mettre en relation des systèmes de relation grâce à des configurations spécifiques de médiations » (op.cit. : 2) assurées quasiment par l'enseignant. Les médiations en questions renvoient à l'ensemble des procédures mises en œuvre par l'enseignant afin d'apporter les étayages nécessaires aux élèves dans le cadre de leur apprentissage. Cette conception des médiations les inscrit dans un contexte pédagogique lié au 
modèle socioconstructiviste et confère en même temps au dispositif «le statut d'analyseur des théories implicites de la formation ou de l'apprentissage » (ibid. : 3).

$\mathrm{S}$ 'en tenir aux acteurs, à leurs interrelations, aux objectifs, à la réalité pédagogique et au statut du dispositif, il est possible de considérer les dispositifs d'enseignement et d'évaluation de la compréhension de l'écrit selon une conception systémique qui permet de les analyser, de les décrire en tant que situations complexes et de relever les dysfonctionnements s'ils existent (Unesco, 1981).

Ces situations qualifiées de complexes combinent plusieurs paramètres dont il est nécessaire de saisir leur nature ainsi que leur mise en relation. Ils seront présentés dans ce qui suit dans la perspective d'exposer les composants du dispositif pédagogique.

\section{Les composantes du dispositif pédagogique}

Les situations complexes auxquelles renvoie le dispositif pédagogique ont tendance à combiner plusieurs paramètres (Montandon, 2002: 14). Ils touchent « aux pratiques pédagogiques diversifiées, qui mobilisent travail individuel, travail en petits groupes ou en grand groupe, diversification des supports, analyse des stratégies d'apprentissages, en relation avec les modalités mises en œuvre, conditions de facilitations des transferts des apprentissages en fonction des caractéristiques spécifiques de la situation et la nature des interventions des enseignants, recourent à des dispositifs de remédiation en fonction des données fournies par l'évaluation dynamique et formative, etc.»(ibid.). Les paramètres énoncés ci-dessus et qui constituent les composants du dispositif peuvent servir de critères d'analyse afin d'approcher l'enseignement de la compréhension de l'écrit en contexte algérien. Les critères d'analyse, selon la précédente citation, recouvrent d'une manière générale les pratiques enseignantes avec ce qu'elles impliquent comme moyens (les supports, les méthodes, les situations d'enseignement et d'évaluation) et les activités des élèves avec ce qu'elles engendrent comme mises en œuvre (déploiement de processus et de stratégies) afin d'assurer le transfert des apprentissages.

Les pratiques enseignantes ainsi que les activités des élèves interagissent dans le cadre de situations pédagogiques dont l'objectif final est la compréhension de l'écrit. Considérées en tant que processus de production, les situations pédagogiques acquièrent le statut de système ayant une sortie et une entrée. L'output ou le produit (Unesco, ibid. : 10), qui est la compréhension de l'écrit (en termes de compétences), est le résultat d'un processus d'enseignement-apprentissage et d'évaluation entre l'enseignant, les élèves, les ressources ainsi que les contraintes (ibid.). L'input ou l'entrée (ibid. : 11), qui renvoie aux élèves, se présente en tant qu'entité déterminante pour l'élaboration du dispositif de formation. Celui-ci obéit aux transformations que vont connaître les élèves. (ibid.). C'est pourquoi Cardinet (1982, cité par Audigier, Crahay et Dolz, 2006) insiste sur le fait que la progression du dispositif de formation doit être établie selon une logique relative au cheminement de l'apprenant pour atteindre les compétences visées. Dans cette optique, il déclare que « l'objectif terminal ne définit nullement le cheminement qui permet d'atteindre la compétence visée. Le dispositif de formation ne peut pas découler de l'analyse de la situation cible, ni même de l'étude des réponses attendues du sujet. Le dispositif de formation serait plutôt à fonder sur une étude (...) des démarches du sujet qui s'approprie la compétence visée. » (ibid. : 13).

À partir de cette déclaration, il est clair qu'il est question d'un déplacement dans les objets d'enseignement - apprentissage et que « leur organisation dépend d'un cadre de référence » (Audigier, Crahay et Dolz, op.cit.) plus large dont il est nécessaire de retracer les contours. Cette entreprise permettra, particulièrement, d'approcher ces objets de manière rigoureuse et d'approcher, généralement, la réalité de la compréhension de l'écrit en contexte scolaire algérien.

XLinguae, Volume 11 Issue 1XL, January 2018, ISSN 1337-8384, eISSN 2453-711X 


\section{Le modèle conceptuel d'analyse et les hypothèses de travail}

En adoptant une démarche systémique afin de décrire la réalité de l'enseignement de la compréhension de l'écrit en contexte algérien, l'idée est de parvenir à dégager un modèle conceptuel d'analyse ayant pour entrées les composants du système identifiées en dessus et mises en relation avec les concepts théoriques mobilisés dans le cadre de ce travail de recherche.

Pour une structuration cohérente (Quivy et Van Campenhoudt, 2011) de ce modèle, il est nécessaire de présenter brièvement notre problématique ainsi que notre question de départ.

La compréhension de l'écrit, en dépit de l'abondante littérature dont elle a fait objet depuis ces dernières décennies, demeure au cœur des préoccupations des chercheurs, des praticiens ainsi que des décideurs politiques. L'accroissement de son intérêt est légitime au regard de son influence sur les niveaux de littératies (Pierre, 1994) déterminants pour un fonctionnement social et planétaire. Néanmoins, ces niveaux connaissent un rehaussement de leurs standards à travers le monde, imposé prioritairement par les exigences de la mondialisation et par l'ère des nouvelles technologies. L'Algérie, par rapport à ces défis externes qui à leur tour imposent de défis internes relatifs à la qualité de la formation (Référentiel général des programmes, 2009), entreprend des réformes curriculaires concernant l'enseignement du français au primaire de manière générale et l'enseignement de la compréhension de textes en $5 \mathrm{e}$ année de primaire de manière particulière. Ainsi, notre recherche se donne pour objectif de rendre compte de cet enseignement dans le cadre de ce nouveau contexte institutionnel ainsi que dans le cadre des avancées disciplinaires du domaine. Les théories et les modèles mobilisés dans le cadre théorique de référence et qui s'inscrivent dans plusieurs domaines (la psycholinguistique du traitement de texte, la psychologie de l'apprentissage, avec la didactique de l'écrit en FLM, les sciences de l'éducation, etc.) gravitent exclusivement autour de trois concepts dont l'opérationnalisation permettra d'appréhender la compréhension de l'écrit à l'ère de la réforme de l'école algérienne. Il s'agit de la compréhension de l'écrit en tant qu'activité langagière, de son enseignement-apprentissage et de son évaluation.

La revue de la littérature a permis dans un premier temps de décrire le comportement d'un lecteur en quête de sens. Cette description est parvenue à travers plusieurs modélisations (van Dijk et Kintsch, 1983 ; Kintsch, 1988 ; 1998 ; Snow, 2002 ; Giasson, 1990 ; 2012) à dégager les facteurs qui lui sont inhérents (processus, stratégies, connaissances antérieures, etc.) ainsi que ceux qui lui sont externes (contexte socio-culturel, contexte familial, etc.). Cette entité centrale dans l'activité de compréhension correspond spécifiquement, dans le cadre du modèle systémique, à l'entrée (l'input), représentée par les élèves.

Dans un second temps, l'état de la recherche met en évidence le caractère indissociable de l'enseignement de la compréhension de son évaluation au regard de la dialectique qui s'instaure entre les deux concepts dans le cadre d'un processus d'acquisition des mécanismes de la compréhension (stratégies, processus, connaissances antérieures, etc.) et de construction du sens. Ce qui confère à ce processus de production le rôle de l'output du dispositif pédagogique. Suite à ces premières attributions ainsi qu'à leurs caractéristiques explicitées dans les composantes du système, il est possible d'esquisser les premiers contours du modèle comme suit : 


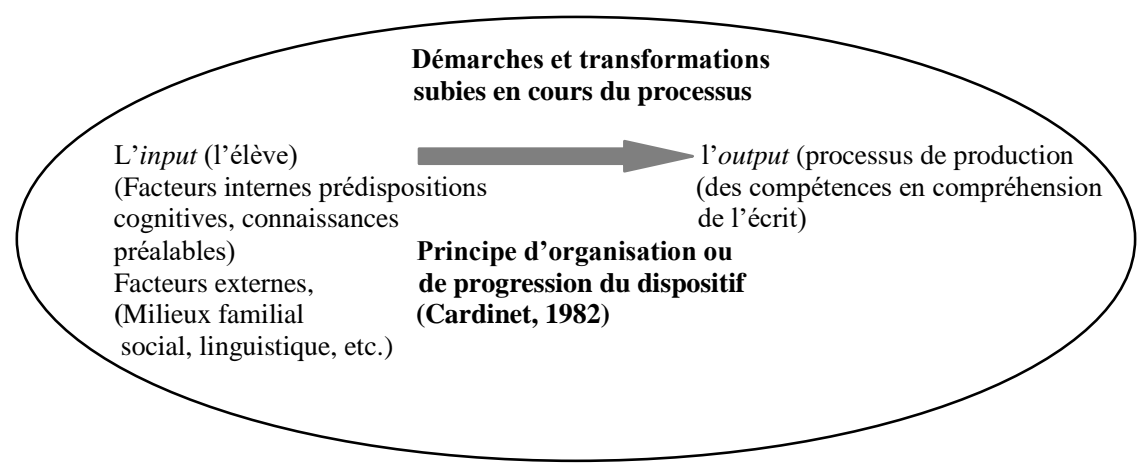

Environnement (Contexte institutionnel et sociolinguistique)

Figure 1 : Les principes organisateurs du dispositif d'enseignement et d'évaluation de la compréhension de l'écrit

Ces premières distributions mettent l'accent sur le principe organisateur du dispositif, ancré dans un environnement spécifique (institutionnel, sociolinguistique) et fondé sur les différentes transformations que connaîtra l'élève lors de ses apprentissages ainsi que sur les différents transferts qu'il effectuera. Ces transformations comme l'a signalé précédemment Cardinet (voir Audigier, Crahay et Dolz, 2006), concernent les démarches conduisant à l'atteinte de la compétence visée.

Ainsi, le dispositif de formation devrait avoir pour toile de fond théorique permettant de planifier sa progression, l'enseignement stratégique de la compréhension de l'écrit (la conceptualisation des étapes de l'enseignement stratégique selon Falardeau et Gagné, 2012), en tant que modèle éclairant l'évolution des compétences à partir de leurs composantes, en l'occurrence les stratégies (la catégorisation des stratégies selon Deschênes, 1991 et Lima, Bianco et Sylvester, 2006).

Une fois le principe qui détermine le cheminement de la progression du dispositif pédagogique désigné, il est question maintenant de relier les autres composantes du système avec la réalité théorique de la compréhension de l'écrit. Ces composantes touchent l'enseignant qui entre en contact avec l'élève dans le cadre du processus d'enseignement-évaluation. Ce processus de production est sous-tendu par le socioconstructivisme en tant que régisseur des relations entre les acteurs du système et en tant que cadre de référence organisant l'objet d'enseignement - évaluation de la compréhension de l'écrit (l'enseignement de stratégies au moyen de l'enseignement collaboratif, de situations - problèmes et de conflits sociocognitifs).

En termes d'interrelations entre acteurs, l'enseignant est concerné par la mise en œuvre de pratiques supposées conduire les élèves à l'acquisition de compétences de compréhension de l'écrit. Dans le cadre d'un dispositif soumis à une progression qui a pour référence théorique l'enseignement stratégique, les pratiques enseignantes, afin d'atteindre les objectifs et les finalités (le principe d'autonomie) tracés par le système éducatif, sont balisées dans un cadre formel bien déterminé.

Celui-ci est du ressort de l'institution qui le diffuse sous forme de pratiques prescrites afin de servir de guide pour l'ensemble des acteurs du système éducatif.

De manière générale, les enseignants sont concernés plus que d'autres acteurs par ce que Goigoux appelle «prescription » et qu'elle considère comme étant : « tout ce que l'institution scolaire définit et communique au professeur pour l'aider à concevoir, à organiser et à réaliser son travail : les programmes d'enseignement et autres instructions officielles, les lois et règlements de la fonction publique d'État, 
l'évaluation du travail enseignant réalisé par les inspecteurs de l'Éducation nationale, l'évaluation des acquisitions des élèves, etc. » (Goigoux, 2007 : 57).

Ces pratiques prescrites sont également accompagnées de directives d'ordre méthodologique et que reprennent les composantes du système relatives aux supports (les textes et les zones de difficultés, établies sous forme de catégories par Joole, 2008), aux méthodes, aux situations d'enseignement, aux situations d'évaluation, etc.

Partie intégrante de l'enseignement stratégique (Falardeau et Gagné, 2012), l'évaluation est également prise en charge dans le cadre de ces pratiques prescrites et obéit à des fondements théoriques compatibles à ceux de l'enseignement. Cela suppose l'évaluation d'objets en lien avec les démarches ayant conduit à la compétence (stratégies cognitives, métacognitives, autorégulatrices) et des situations - problèmes pour observer l'exercice de cette compétence. Il est également question d'acquisition de critères d'évaluation susceptible de générer des apprentissages.

Cette vision de l'objet attribue à l'évaluation plusieurs fonctions (diagnostique, formative, certificative, autorégulatrice) sur un même continuum qui permet l'observation de la progression des apprentissages ainsi que l'observation des pratiques enseignantes. Au terme de ces mises en relation entre composantes du dispositif pédagogique dans sa conception systémique, et les concepts théoriques relatifs à la compréhension de l'écrit, à son enseignement ainsi qu'à son évaluation, les constituants des dispositifs d'enseignement et d'évaluation de la compréhension de textes se précisent dans un premier temps sur le plan des théories étayant les relations entre acteurs et le processus d'enseignement-apprentissage et évaluation. Cet arrièreplan théorique se présente comme suit :

Les finalités et les objectifs du dispositif

Les acteurs

Les principes organisateurs

Le statut du dispositif

Le contexte ou

la réalité pédagogique

Les finalités et les objectifs du dispositif

Les acteurs

Les principes organisateurs

Le statut du dispositif

Le contexte ou

la réalité pédagogique

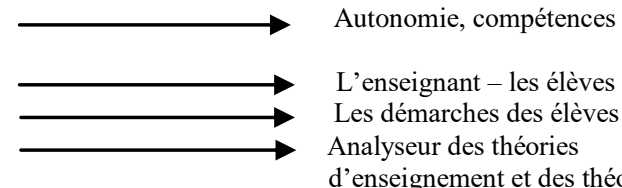

d'enseignement et des théories d'apprentissage

Les médiations (rôle enseignant)

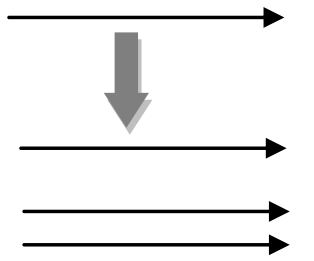

Autonomie en compréhension de l'écrit - Acquisition de Compétences

L'enseignant - les élèves

Progression en fonction du développement des compétences (L'enseignement stratégique)

Les théories d'enseignement apprentissage et d'évaluation de la compréhension de texte (les modèles interactifs, les modèles socioconstructivistes)

La dimension sociale des médiations dans l'enseignement - apprentissage de la compréhension de textes (le caractère transparent des démarches d'apprentissage au moyen d'étayage, d'enseignement collaboratif et de conflits sociocognitifs)

Figure 2 : Les fondements théoriques du dispositif d'enseignement et d'évaluation de la compréhension de l'écrit 
Dans un second temps, les dispositifs d'enseignement et d'évaluation se précisent sur le plan des dimensions concrètes à partir desquelles il est possible d'appréhender la compréhension de l'écrit dans le cadre de la réforme du système éducatif algérien. Celles-ci concernent les pratiques d'enseignement et les pratiques d'évaluation du fait qu'elles sont reconnues comme une composante du dispositif pédagogique, qu' elles émanent d'instances institutionnelles et qu'elles s'inscrivent dans des modèles théoriques appropriés. Ainsi, il est possible de schématiser les concepts de la compréhension de l'écrit et leurs dimensions comme suit :

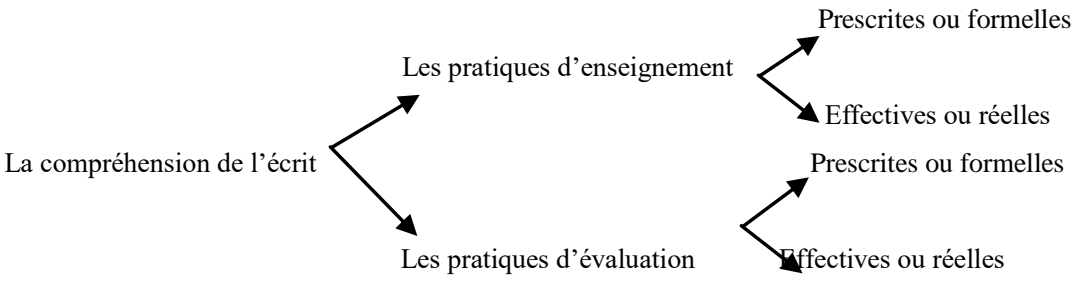

\section{Figure 3 : Les concepts de la compréhension de l'écrit et leurs dimensions}

À partir de cette schématisation, il est clair qu'une approche tangible de la compréhension de l'écrit ne peut s'opérer qu'à travers ces pratiques qui constituent en soi des sources de savoir et que seule l'analyse permet de saisir (Pescheux, 2007). Pour atteindre ces savoirs, il est nécessaire de spécifier la question générale qui s'interroge sur l'enseignement et l'évaluation de la compréhension de l'écrit dispensés en 5 e année de primaire en contexte algérien. La question principale peut se décliner en questions de recherche que voici :

- Quel (s) objet (s) est (sont) mis en perspective par les pratiques prescrites et les pratiques effectives d'enseignement-évaluation de la compréhension de l'écrit en $5 \mathrm{e}$ année de primaire?

- À la lumière de cet (ces) objet (s), quel (s) modèle (s) théorique (s) sous-tend (ent) les pratiques prescrites et les pratiques effectives d'enseignement-évaluation de la compréhension écrite en 5e année de primaire?

- Dans quelle mesure les pratiques prescrites et les pratiques effectives d'enseignement et d'évaluation de la compréhension de l'écrit sont- elles congruentes ou incongruentes?

Ces questionnements aspirent à élucider la problématique de la compréhension de l'écrit à travers son enseignement et son évaluation en interpellant ses objets et ses fondements théoriques à la lumière des pratiques mises en place. À l'égard de cellesci, nous postulons :

- Qu'en enseignement de la compréhension de l'écrit, elles mettent en perspective les compétences de déchiffrage comme préalable aux compétences de compréhension.

- Qu'en lien avec ce qui a été mis en perspective en enseignement, les pratiques d'évaluation mettent en avant dans les épreuves des items qui favorisent une mobilisation plutôt parcellaire qu'interactive, des compétences de la compréhension d'un texte.

- Qu'en dépit de leur détermination par des pratiques prescrites consignées dans un curriculum officiel (Glatthorn, 1987, cité par Audigier, Crahay et Dolz, 2006), les pratiques effectives de l'enseignement-évaluation de la compréhension de l'écrit ou le curriculum effectivement enseigné, demeurent en décalage par rapport à celui-ci. 
La formulation de ces hypothèses permet de constater un élargissement conceptuel vers le curriculum officiel (ibid.) et le curriculum effectivement enseigné (ibid.) dans une perspective comparative. Le but de cette comparaison est de pouvoir s'arrêter sur le degré de mise en œuvre du curriculum recommandé (ibid.) et sur son degré de répercussion sur le curriculum acquis par les élèves (ibid.).

L'introduction du curriculum acquis est justifiée par l'idée qu'il est partie intégrante du processus d'enseignement-évaluation.

$\mathrm{Au}$ vu de l'ampleur de ce que peut recouvrir un curriculum, il est nécessaire de préciser l'usage dont il est fait de ce concept dans le cadre de notre recherche à travers les dimensions retenues.

L'utilisation que nous faisons du concept « curriculum » et qui s'inspire de la théorie de conceptualisation historique du curriculum de Kliebard (1992, cité par Audigier, Crahay et Dolz, 2006), recouvre principalement le « que doit-on enseigner » (ibid. : 122) qui renvoie aux savoirs définis dans le nouveau curriculum en termes de compétences disciplinaires et transversales, et « le comment enseigné ce quoi à qui ?» (ibid.) qui " se réfère à l'analyse de la mise en œuvre du curriculum à travers les pratiques d'enseignement » (Audigier, Crahay et Dolz, ibid.) susceptibles d'assurer la transmission de ce savoir aux élèves. Ainsi, l'usage du concept curriculum s'opèrera dans le but de désigner les pratiques d'enseignement et leur rapport avec les démarches d'apprentissage des élèves ayant pour cadre d'action " la situation qui requiert celui de dispositif » (ibid. : 123).

En effet, le dispositif en tant que cadre intégrateur, englobe des situations. Montandon précise ce rapport qui existe entre dispositif et situation en signalant que celle-ci « est ponctuelle, mise en place à un moment déterminé » (2002: 31) alors que «le dispositif intègre la dimension temporelle, et doit être pensé comme un ensemble intégrateur des différents paramètres convoqués dans toute situation d'apprentissage interactif, et permettant d'opérationnaliser les processus d'apprentissage des individus. » (ibid.).

La clarification des rapports entre dispositif et situation est indispensable pour notre observation. Cette observation portera sur les paramètres du dispositif, mais qui seront analysés dans le cadre de situations d'enseignement - évaluation de la compréhension de l'écrit sélectionnées. L'observation de situations sélectionnées s'explique par le fait qu'il est impossible de rendre compte de toutes les situations que peut contenir un dispositif pédagogique à cause de son caractère « dynamique et instable, susceptible d'évoluer au cours du processus de formation et d'apprentissage » (ibid. : 35).

Suite aux dimensions et aux indicateurs retenus, il est possible de schématiser les pratiques en rapport avec le concept de curriculum et le modèle conceptuel qui synthétise les critères d'analyse retenus dans les figures 4 et 5 .

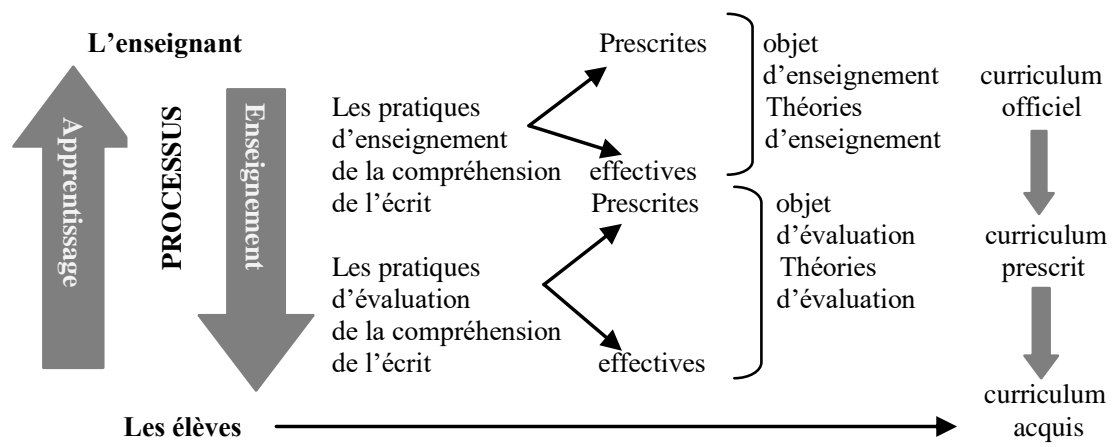

Figure 4 : Les indicateurs retenus pour le concept de la compréhension de l'écrit 


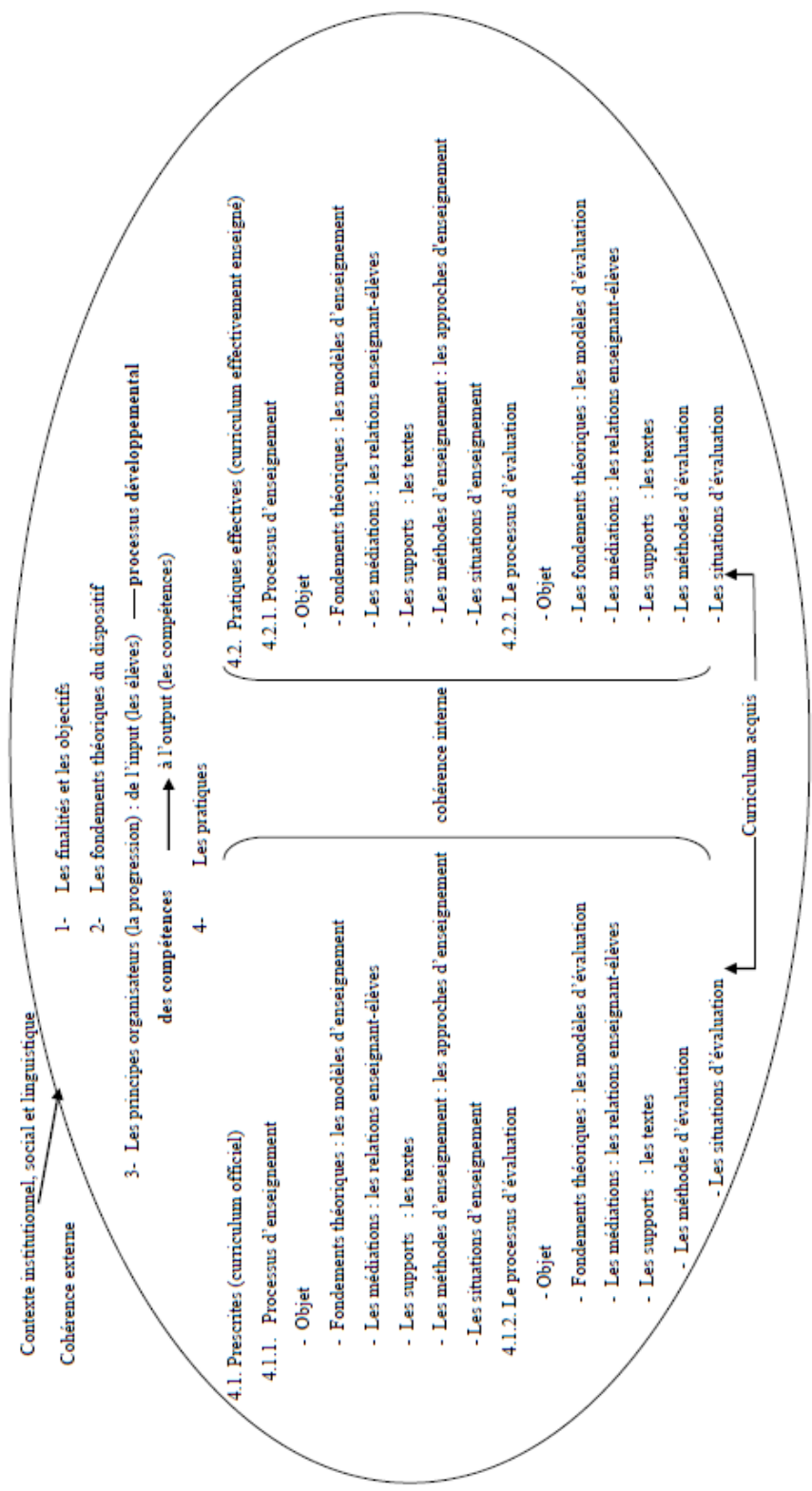

Figure 5 : Les entrées du dispositif pédagogique d'enseignement et d'évaluation de la compréhension de l'écrit

XLinguae, Volume 11 Issue 1XL, January 2018, ISSN 1337-8384, eISSN 2453-711X 145 
Les entrées présentes dans le modèle conceptuel d'analyse de la compréhension de l'écrit (Figure 5) ont permis d'analyser le corpus recueilli dans le cadre de la recherche doctorale. Celui-ci comprend des données écrites et des données orales analysées en fonction des quatre dimensions des pratiques de la compréhension de l'écrit et des indicateurs qui leur sont liés. Celles-ci sont réparties comme suit : 


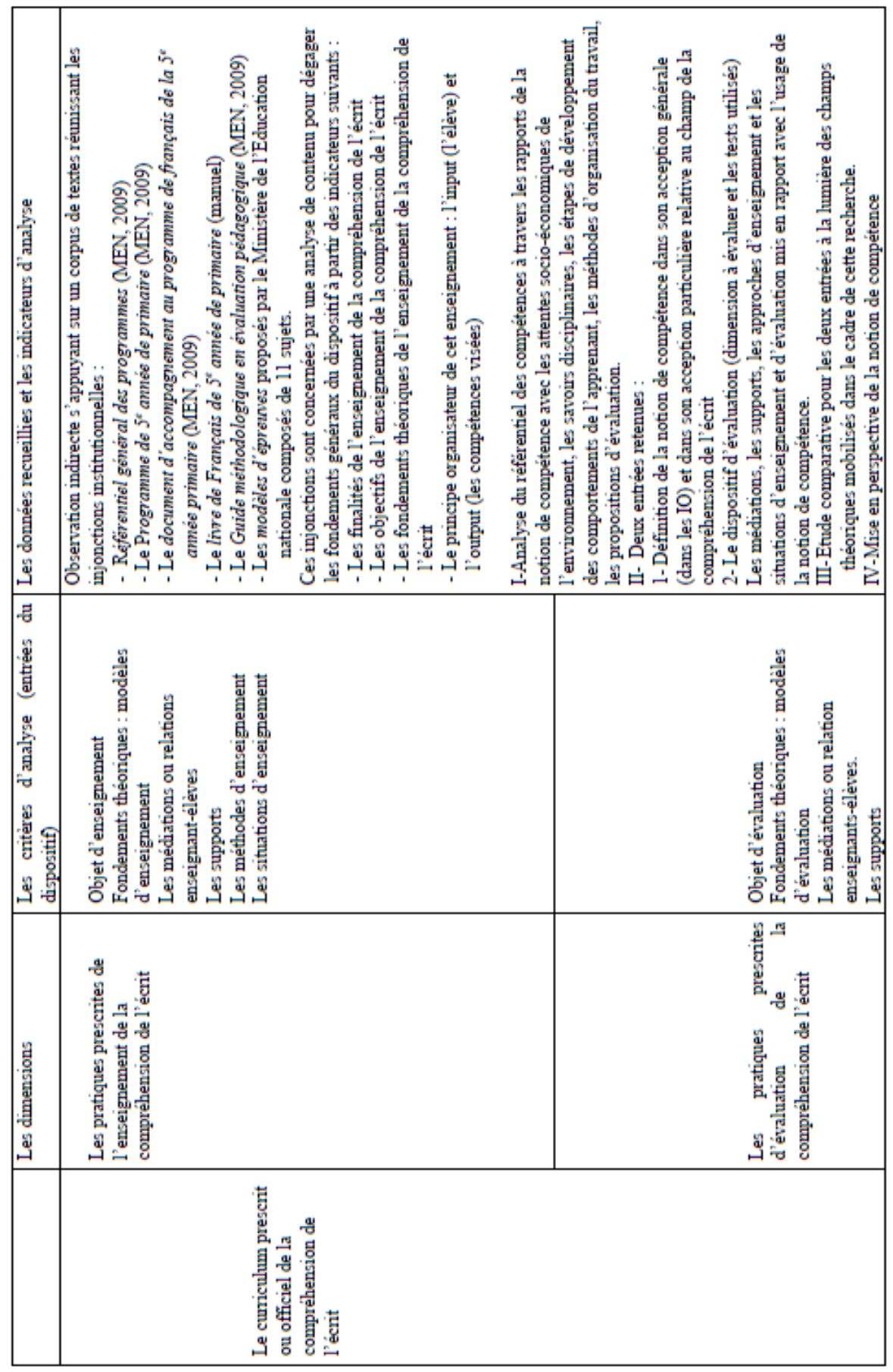

XLinguae, Volume 11 Issue 1XL, January 2018, ISSN 1337-8384, eISSN 2453-711X 


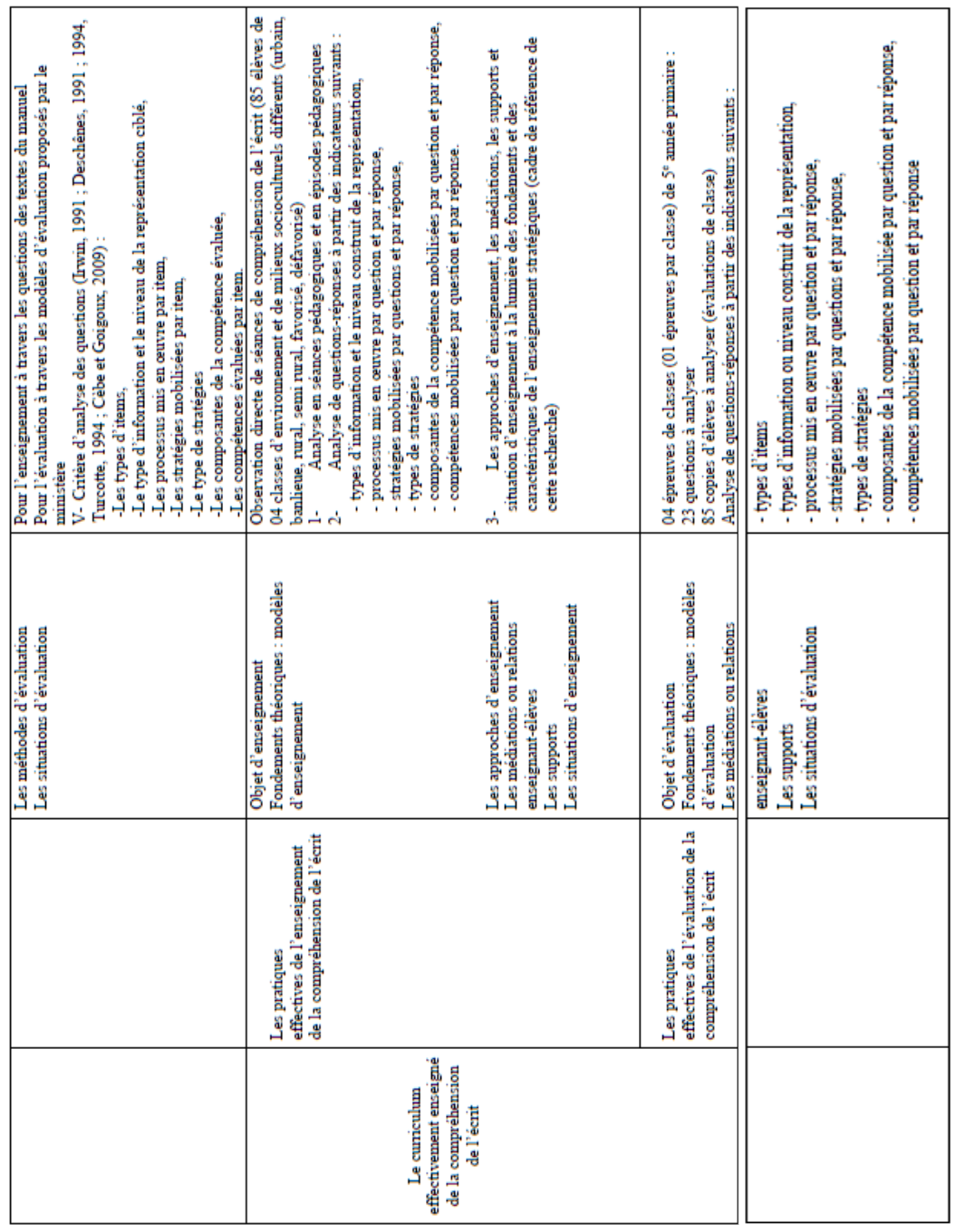

Tableau 1 et 2 : Le protocole de recherche du dispositif d'enseignement et d'évaluation de la compréhension de l'écrit 


\section{Bibliographic references}

AUDIGIER, F. - CRAHAY, M. - DOLZ, J. 2006. Curriculum, enseignement et pilotage. Bruxelles : de boeck.

CARDINET, J. 1982. Compétences, capacités, indicateurs : quel statut scientifique ? Texte présenté aux rencontres sur l'évaluation. Lyon : Centre d'études pédagogiques pour l'expérimentation et le conseil.

DESCHENES, A-J. 1991. La lecture une activité stratégique. In : Les entretiens Nathan sur la lecture. Paris : Nathan, pp.29-49

FALARDEAU, E. - GAGNE, J-C. 2012. L'enseignement explicite des stratégies de lecture : des pratiques fondées par la recherche. In : Enjeux, n.83, pp. 91-120.

GIASSON, J. 1990. La compréhension de l'écrit. Bruxelles : de boeck.

GIASSON, J. 2012. La lecture. Apprentissage et difficultés. Bruxelles : De Boeck.

GOIGOUX, R. 2007. Un modèle d'analyse de l'activité des enseignants. In : et Didactique, vol.1, n. 3, pp.47-69.

JOOL, P. 2008. Comprendre les textes écrits. Paris : Retz, CRDP, l'Académie de Versailles.

KINTSCH, W. 1988. The role of knowledge. In: discourse comprehension: A construction integration model. Psychological Review, n. 95, pp. 163-182.

KINTSCH, W. 1998. Comprehension, a Paradigm for Cognition. New York: Cambridge University Press.

LIMA, L. - SYLVESTRE, E. - BIANCO, M. 2006. Lectures partagées et acquisition de stratégies de compréhension au cycle 3. In : DESSUS, P. et GRENTAZ, E. (Éds), Apprentissages et enseignement. Sciences cognitives et éducation. Paris : Dunod, pp.25-42.

MINISTERE DE L'EDUCATION NATIONALE. 2009. Référentiel général des programmes. Alger: ONPS.

MONTANDON, C. 2002. Approche systémique des dispositifs pédagogiques. Enjeux et méthodes. Paris : l'Harmattan.

PECHEUX, M. 2007. Analyse de pratique enseignante en FLE/S. Mémento pour une ergonomie didactique en FLE. Paris : L'Harmattan.

PIERRE, R. 1994. Savoir lire aujourd'hui : de la définition à l'évaluation du savoirlire in J-Y. BOYER, J-P. DIONNE, P. RAYMOND, Évaluer le savoir- lire. Montréal : Les Éditions logiques, pp. 275-315

QUIVY, R. - VAN CAMPENHOUDT, L. 2006. Manuel de recherche en sciences sociales. Paris : Dunod.

UNESCO. 1981. L'éducateur et l'approche systémique. Manuel pour améliorer la pratique de l'éducation. Paris : Presses Universitaires de France.

VAN DIJK, T.A. - KINTSCH, W. 1983. Strategies of discourse comprehension. New York : Academic Press.

SNOW, C. 2002. Reading for understanding: Toward an R et D program in reading comprehension. Santa Monica: Rand.

Words: 4573

Characters: 36323 (20, 18 standard pages)

Dr. Zineb Haroun, PhD.

Department of Letters and French Language

Faculty of Arts and Languages

University the Mentouri Brothers, Constantine 1

Algeria

magister50@gmail.com

XLinguae, Volume 11 Issue 1XL, January 2018, ISSN 1337-8384, eISSN 2453-711X 